\title{
Composition, cytotoxicity and antioxidant activities of polyphenols in the leaves of star anise (Illicium verum Hook. f.)
}

\author{
Ying Wang ${ }^{\mathrm{a}}$, Tao Liu ${ }^{\mathrm{a}}$, Ming-Fen Li ${ }^{\mathrm{a}}$, Yun-Shu Yang ${ }^{\mathrm{a}}$, Rong Li ${ }^{\mathrm{a}, *}$, Jin Tan ${ }^{\mathrm{a}}$, Shu-Hua Tang ${ }^{\mathrm{a}}$, \\ Zi-Tao Jiang ${ }^{\mathrm{a}, \mathrm{b}, *}$ \\ a Tianjin Key Laboratory of Food Biotechnology, College of Biotechnology and Food Science, \\ Tianjin University of Commerce, Tianjin 300134 China \\ b School of Food Engineering, Tianjin Tianshi College, Tianjin 301700 China \\ *Corresponding authors, e-mail: ztjiang@yahoo.com, lirong@tjcu.edu.cn
}

Received 12 Aug 2019

Accepted 21 Oct 2019

\begin{abstract}
The polyphenols in the leaves of star anise (Illicium verum Hook. f.) were qualitatively and quantitatively determined by LC-Q-TOF-MS and LC-MS/MS. Twelve flavones and a phenolic acid were identified, mainly comprising vitexin-2"-O-rhamnoside ( $8780.12 \mu \mathrm{g} / \mathrm{g}$ ) and vitexin-2"-O-xylopyranoside (575.46 $\mu \mathrm{g} / \mathrm{g}$ ), which have high free radical scavenging abilities and strong lipid peroxidation inhibitory activities. In addition, they could also reduce reactive oxygen species in HT-29 cells without cytotoxicity.
\end{abstract}

KEYWORDS: Illicium verum Hook. f., polyphenols, vitexin-2"-O-rhamnoside, antioxidant activities, intracellular antioxidation

\section{INTRODUCTION}

Star anise (Illicium verum Hook f.) is an economic tree species, belonging to the Illicium genus of the Illiciaceae family and is distributed mainly in Vietnam, Thailand and southern China ${ }^{1}$. Its fruit is a famous spice. Seven compounds comprising flavones, such as naringenin-4"-O- $\beta$ D-glucopyranoside, quercitin-3-OL-rhamnose, and benzeneethanol-4-O- $\beta$-D-glucopyranoside were isolated and were obtained from I. verum plants for the first time ${ }^{2}$. In addition, Yi et al separated three flavone glycosides from I. verum leaves, including quercetin, kaempferol, and isorhamnetin, by high-performance liquid chromatography ${ }^{3}$. Sy and Brown discovered novel phenylpropanoids and lignans from $I$. verum, including some phenolics ${ }^{4}$. These compounds had significant antioxidant and anti-inflammatory properties ${ }^{5}$. As a by-product, I. verum leaves are abundant, but poorly utilized, resulting in wasted resources.

To the best of our knowledge, there has been no systematic report on the polyphenols of I. verum leaves and their antioxidant activities. In the present study, polyphenols in I. verum leaves were identified and quantified via LC-Q-TOF-MS and LCMS/MS. Antioxidant activities were determined by chemical and cellular modelling methods. The relationship between the structure of the polyphenol and the antioxidant activity was also evaluated.

\section{MATERIALS AND METHODS}

\section{Plant Materials}

The fresh I. verum leaves were collected from Hechi City, Guangxi Province, China on September 2014 and verified by Professor Xie Junbo (School of Chinese Materia Medica, Tianjin University of Traditional Chinese Medicine, Tianjin, China).

\section{Extraction, Purification, and Analysis of polyphenols from $I$. verum leaves}

The polyphenols were extracted according to the following process. $5 \mathrm{~g}$ of ground leaf powder and $130 \mathrm{ml}$ of $67 \%$ ethanol $(\mathrm{V}: \mathrm{V})$ were mixed using microwave-assisted extraction. The conditions were as follows: extraction time $5 \mathrm{~min}$, microwave power $500 \mathrm{~W}$ and extraction temperature $75^{\circ} \mathrm{C}$. The resulting extract was vacuum filtered and further extracted three times with isopycnic petroleum ether to remove the fat-soluble components. The extract was concentrated and freeze-dried. According to our previous study ${ }^{6}$, this crude extract was purified and separated by macroporous adsorption resin D101 and preparative chromatography (Grace 
Reveleris $^{\mathrm{TM}}$ system, Alltech, US), then analysed qualitatively and quantitatively by LC-MS/MS.

\section{Validation of assay}

The limit of detection (LOD) was detected by measuring the signal-to-noise ratio ( $\mathrm{S} / \mathrm{N}$ ratio) 3 times, while the limit of quantification was detected by measuring the $\mathrm{S} / \mathrm{N}$ ratio 10 times. The accuracy of the method was evaluated by analysing six replicates of mixed standard solutions or samples and expressed as the relative standard deviation (RSD). The accuracy of the method is assessed by a recovery test. An appropriate amount of the mixed standard solution was added to the purified polyphenol extract solution. Subsequently, the mixture was processed and analysed according to the method described by Yang et $\mathrm{al}^{6}$.

\section{Antioxidant activity analyses}

Various aspects of antioxidant activities were evaluated by chemical methods ${ }^{7-9}$, including superoxide anion radical scavenging activities, the lipid peroxidation inhibition activities, the hydroxyl radical scavenging activities, the total antioxidant activities and the 1,1-diphenyl-2-picrylhydrazyl (DPPH) free radical scavenging activities. Compared to chemical methods, the cell model method has a better biological correlation and could more accurately predict the antioxidant activity of antioxidants in the human body. Hence human colon cancer cells (HT-29 cells) were chosen to simulate an in vivo environment to study the intracellular antioxidant activities of polyphenols. HT-29 cells were obtained from the Institute of Biochemistry and Cell Biology (Shanghai, China) and there was no mycoplasma contamination according to the mycoplasma test method $^{10}$. Cell viability and intracellular antioxidation were measured according to the previous method described by both Schantz et $\mathrm{al}^{11}$ and Yang et $\mathrm{al}^{6}$. The intracellular antioxidant activity (CAA) was calculated according to the following equation: $\mathrm{CAA}(\%)=100-\left(\int \mathrm{SA} / \int \mathrm{CA}\right) \times 100$, fluorescence intensities were measured every 5 minutes during the 1-h incubation time, $\int \mathrm{SA}$ and $\int \mathrm{CA}$ are the integral areas of the relative fluorescence intensity of the sample and control ${ }^{11,16}$.

\section{Statistical Analysis}

Student's $t$-test was performed to compare the differences between total polyphenols and vitexin-2"O-rhamnoside at the same concentration in the antioxidation assay. Cell viability and intracellular antioxidation were compared by one-way ANOVA.

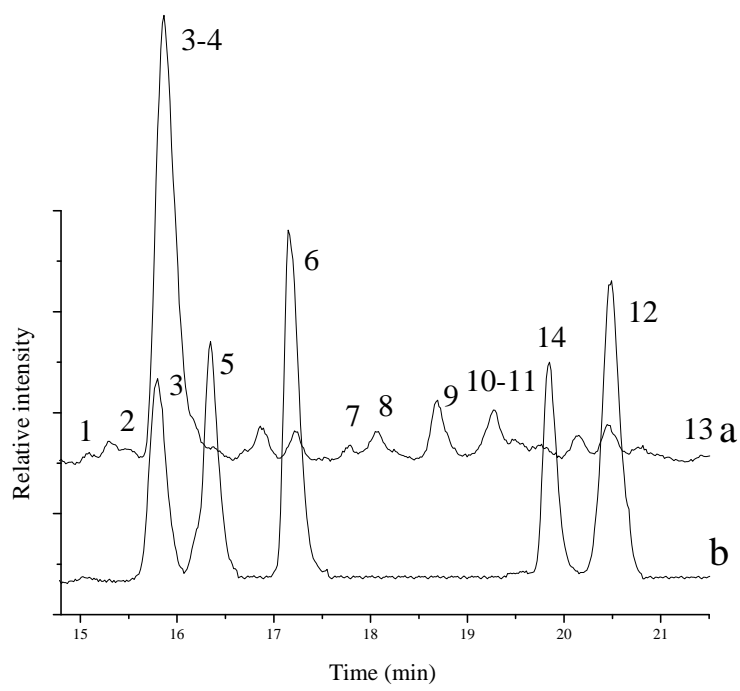

Fig. 1 Total ion chromatograms of (a) the purified polyphenols extracted from I. verum leaves and (b) the mixture of reference substances. Peak numbers refer to Table 1.

All statistical analyses were performed by SPSS (version 16.0). All results were based on three replicates indicated as mean values and standard deviations.

\section{RESULTS AND DISCUSSION}

\section{Qualitative Analysis}

Under the same analytical conditions, the retention time, molecular ion and $\mathrm{MS}^{2}$ spectrum (peaks 3 , $5,6,7$, and 12) identified five components with standard substances (Fig. 1a and Fig. 1b). The other peaks in Fig. 1a were determined by MS/MS and comparison with Refs. 12-20. Twelve flavones and a phenolic acid have been identified and the results are presented in Table 1. The content and variety of the identified polyphenols were different from previously reported data ${ }^{2-4}$, which could be explained by the different origins and sources of the collected plant. The collected plant can be age or tissue-specific. The other eight components that do not contain available standard substances could be distinguished by relevant pieces of literature, but they deserve further study once standards are available.

\section{Quantitative Analysis}

Five different concentrations of standard substances were analysed by LC-MS/MS for the quantification of polyphenols. Table 2 shows the calibration equations of the five components and the contents 
Table 1 Retention times and fragment ions of components and standard substances in $I$. verum leaves.

\begin{tabular}{|c|c|c|c|c|c|c|}
\hline \multirow{2}{*}{ Peak } & \multirow{2}{*}{ Compound } & \multirow{2}{*}{$\begin{array}{c}{[\mathrm{M}-\mathrm{H}]^{-}} \\
(\mathrm{m} / \mathrm{z})\end{array}$} & \multicolumn{2}{|c|}{ LC-Q-TOF-MS } & \multicolumn{2}{|c|}{ LC-MS/MS } \\
\hline & & & $\mathrm{RT}(\min )$ & $\mathrm{MS}^{2}(\mathrm{~m} / \mathrm{z})$ & $\mathrm{RT}(\min )$ & $\mathrm{MS}^{2}(\mathrm{~m} / \mathrm{z})$ \\
\hline 1 & Vitexin-4"-O-glucoside & 593 & 15.09 & 413,293 & 10.80 & 413,293 \\
\hline 2 & P-coumaroylquinic acids & 337 & 15.41 & 191,173 & 10.85 & 191,173 \\
\hline 3 & Vitexin-2"-O-rhamnoside & 577 & 15.58 & $413,311,293$ & 11.86 & $413,311,293$ \\
\hline 4 & Vitexin-2"-O-xylopyranoside & 563 & 16.00 & 413, 311, 293 & 12.08 & 413, 311, 293 \\
\hline 5 & Rutin & 609 & 16.46 & 301300 & 13.69 & 301,300 \\
\hline 6 & Vitexin & 431 & 17.24 & $311,283,161$ & 12.41 & $341,311,283$ \\
\hline 7 & Hyperoside & 463 & 17.83 & $300,271,255$ & 13.96 & $300,271,255$ \\
\hline 8 & Isorhamnetin-3-O-rutinoside & 623 & 18.03 & $315,314,299$ & 14.02 & $315,314,299$ \\
\hline 9 & Kaempferol-7-glucoside & 447 & 18.94 & $285,255,227$ & 15.39 & $285,255,227$ \\
\hline 10 & Quercetin-3-O-xylopyranoside & 433 & 19.53 & $300,271,255$ & 14.87 & $300,271,255$ \\
\hline 11 & Isorhamnetin-3-O-glucoside & 477 & 19.55 & $314,300,271$ & 15.66 & 314,271 \\
\hline 12 & Genistin & 431 & 20.42 & 268 & 15.18 & 268 \\
\hline 13 & Kaempferol-3-O-arabinoside & 417 & 21.46 & $285,255,227$ & 16.33 & $285,255,227$ \\
\hline 14 & Avicularin & 433 & 20.08 & $300,271,255$ & 15.04 & $300,271,255$ \\
\hline
\end{tabular}

Table 2 Quantification results of components in I. verum leaves.

\begin{tabular}{llccccc}
\hline Peak & Compound & Calibration curve & $\begin{array}{c}\text { Linear range } \\
(\mu \mathrm{g} / \mathrm{ml})\end{array}$ & $\begin{array}{c}\mathrm{R}^{2} \\
(\mu \mathrm{g} / \mathrm{g})\end{array}$ & $\begin{array}{c}\text { Content }^{\mathrm{a}} \\
(\%)\end{array}$ & $\begin{array}{c}\text { Recovery }^{\mathrm{a}} \\
(\%)\end{array}$ \\
\hline 1 & vitexin-4"-O-glucoside & - & - & - & $71.4 \pm 2.0^{\mathrm{b}}$ & - \\
2 & 3-p-coumaroylquinic acids & - & - & - & $112.6 \pm 3.3^{\mathrm{b}}$ & - \\
3 & vitexin-2"-O-rhamnoside & $Y=-42449.72+19565.48 X$ & $0.1-10.0$ & 0.998 & $8780 \pm 185$ & $105.2 \pm 3.2$ \\
4 & vitexin-2"-O-xylopyranoside & - & - & - & $575 \pm 13^{\mathrm{b}}$ & - \\
5 & rutin & $Y=-3392.61+17337.44 X$ & $0.05-6.0$ & 0.998 & $38.8 \pm 1.7$ & $107.3 \pm 2.6$ \\
6 & vitexin & $Y=108442.17+11324.55 X$ & $0.1-4.0$ & 0.995 & $20.7 \pm 0.6$ & $103.8 \pm 3.4$ \\
7 & hyperoside & $Y=169852.67+15585.71 X$ & $0.05-6.0$ & 0.999 & $10.7 \pm 0.1$ & $98.7 \pm 1.3$ \\
8 & isorhamnetin-3-O-rutinoside & - & - & - & $23.0 \pm 0.3^{\mathrm{b}}$ & - \\
9 & kaempferol-7-glucoside & - & - & - & $7.3 \pm 0.3^{\mathrm{b}}$ & - \\
10 & quercetin-3-O-xylopyranoside & - & - & - & $43.8 \pm 1.3^{\mathrm{b}}$ & - \\
11 & isorhamnetin-3-O-glucoside & - & - & - & $10.5 \pm 0.2^{\mathrm{b}}$ & - \\
12 & genistin & $Y=71095.55+18714.78 X$ & $0.05-6.0$ & 0.999 & $4.4 \pm 0.1$ & $93.8 \pm 3.8$ \\
13 & kaempferol-3-O-arabinoside & - & - & - & $9.2 \pm 0.2^{\mathrm{b}}$ & - \\
\hline
\end{tabular}

${ }^{\text {a }}$ Mean \pm SD of three replicates;

${ }^{\mathrm{b}}$ Expressed in the equivalents of vitexin.

of 13 components. Although vitexin has a structure similar to the eight non-standard components available and the eight-component content has been expressed as equivalents of the quantitative method of vitexin equivalence, they deserve further study once standards become available.

\section{Validation of assay}

LOD and the limit of quantitation (LOQ) were 0.10$14.80 \mathrm{ng} / \mathrm{ml}$ and $0.33-49.41 \mathrm{ng} / \mathrm{ml}$, respectively. Correlation coefficients $\left(\mathrm{R}^{2}\right)$, recoveries and RSD values indicating satisfactory reliability and accuracy of the current method are presented in Table 2 .

\section{Separation of extract from $I$. verum leaves}

The polyphenols were separated and collected by macroporous resin and preparative chromatography. The main components accounting for $96.3 \%$ of the total polyphenols were vitexin-2"-O-rhamnoside (90.4\%) and vitexin-2"-O-xylopyranoside (5.9\%). The structures of the two compounds were shown in Fig. 2, illustrating rings A and B.

\section{Antioxidant activity analyses}

All the antioxidant activities measured in this study were concentration-dependent (Fig. 3). In the present study, there was a linear relationship between absorption and antioxidant concentration of 


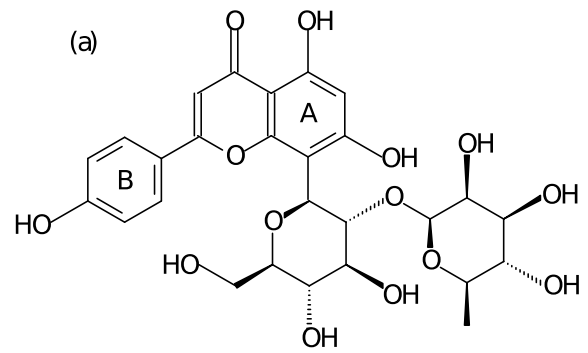<smiles>O=c1cc(-c2ccc(O)cc2)oc2c(C3OC(CO)C(O)C(O)C3OC3OCC(O)C(O)C3O)c(O)cc(O)c12</smiles>

Fig. 2 The structures of (a) vitexin-2"-O-rhamnoside and (b) vitexin-2"-O-xylopyranoside.
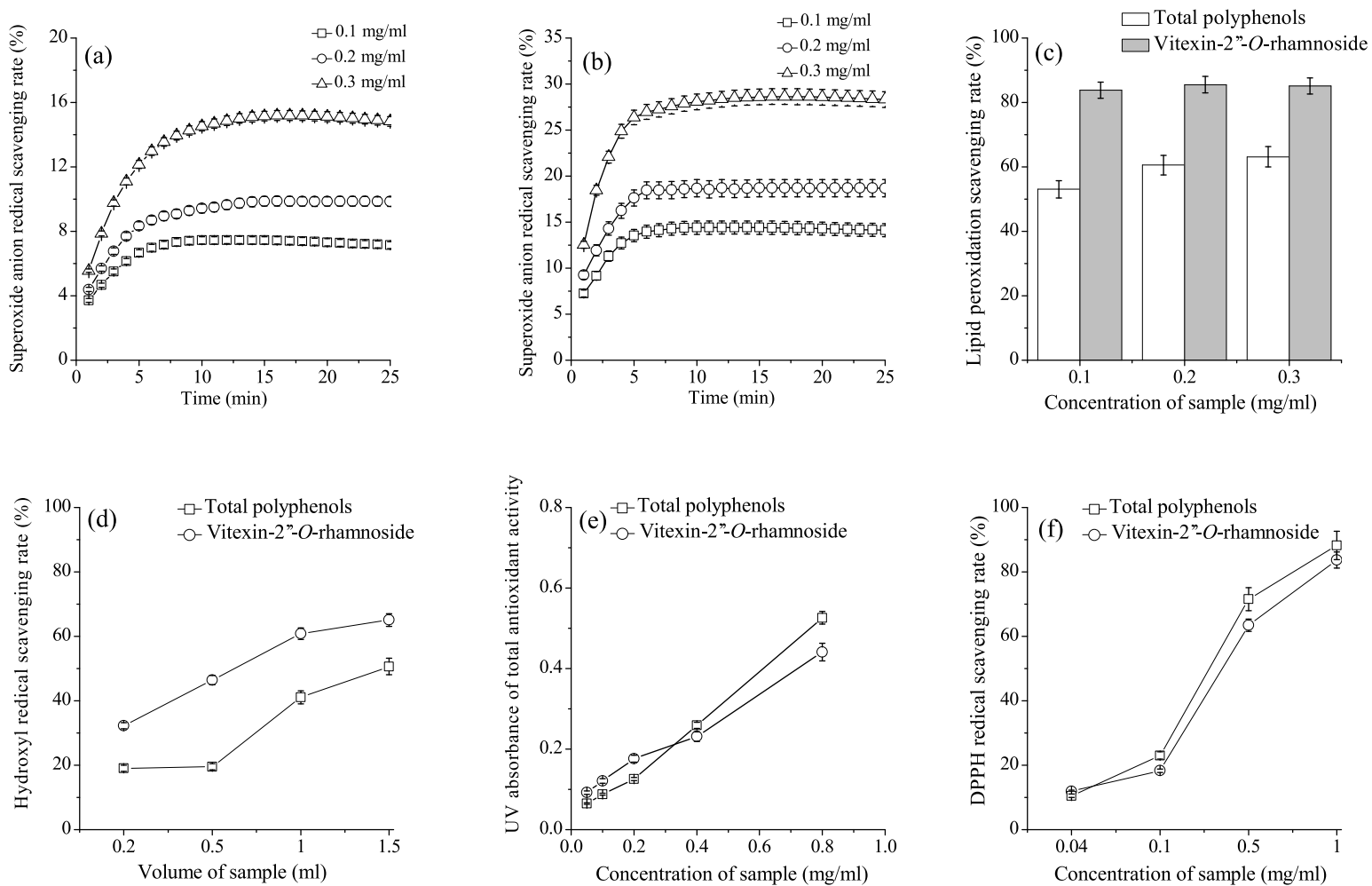

Fig. 3 Superoxide anion scavenging activity of (a) total polyphenols and (b) vitexin-2"-O-rhamnoside; (c) lipid peroxidation inhibition activity, (d) hydroxyl radical scavenging activity, (e) total antioxidant activity, and (f) DPPH radical scavenging activity of both total polyphenols and vitexin-2"-O-rhamnoside. Data are reported as the mean \pm SD of three replicates.

0-3 min for superoxide anion scavenging (Fig. 3a and Fig. 3b). The antioxidant capability of vitexin2"-O-rhamnoside was higher than that of total polyphenols ( $t$-test, $p<0.05$ ), but the two capabilities of vitexin-2"-O-rhamnoside and polyphenols totals were low (Fig. 3a and Fig. 3b). This could be explained by the glycoside groups of vitexin-2"O-rhamnoside which could introduce steric effects, thereby reducing the scavenging activity of superoxide anion radicals ${ }^{21}$.

The effects of the lipid peroxidation inhibitory activity are shown in Fig. 3c the scavenging rate of vitexin-2"-O-rhamnoside was significantly higher than that of total polyphenols ( $t$-test, $p<0.05$ ). This result indicated that vitexin-2"-O-rhamnoside had high scavenging activity even at low concentrations, possibly due to the 4-ketonic group and the 5-hydroxyl group structure in vitexin-2"-Orhamnoside could chelate $\mathrm{Fe}^{2+}$, while the 4-ketonic group also facilitates the formation of more stable free radical intermediates, which reduces the reaction strength ${ }^{22}$.

For hydroxyl radical scavenging (Fig. 3d), vitexin-2"-O-rhamnoside had the highest scaveng- 

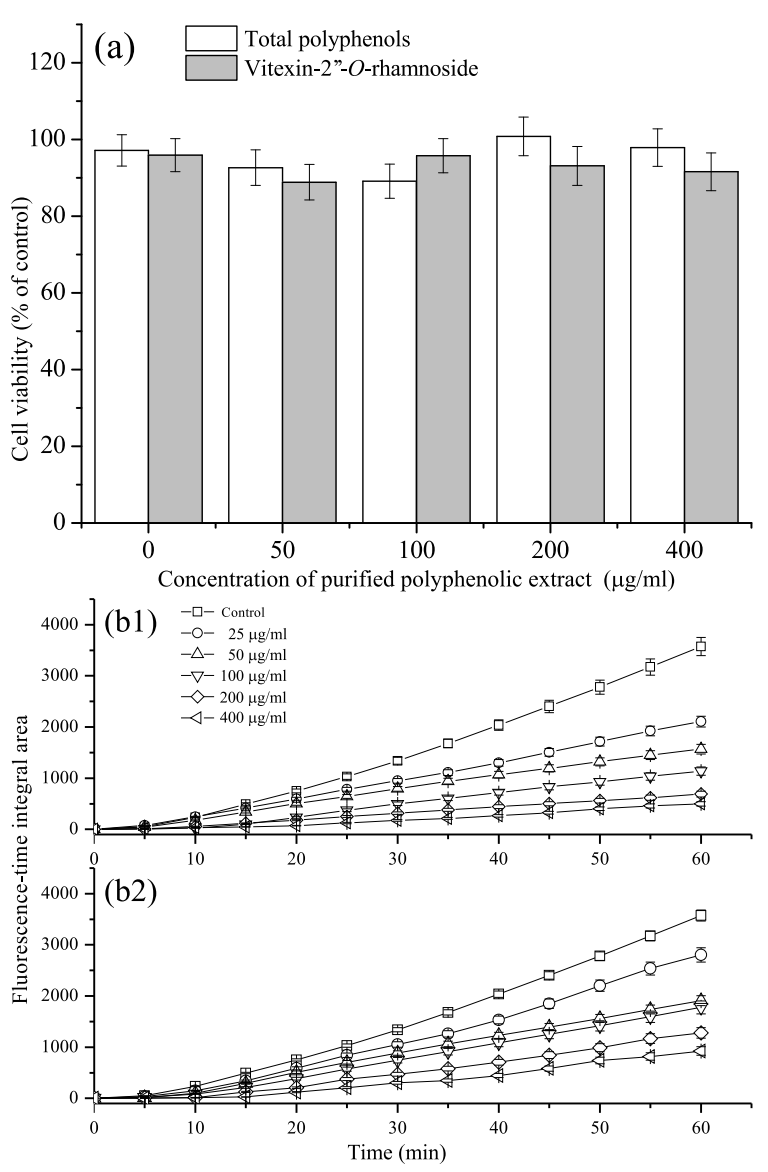

Fig. 4 Effects of total polyphenols and vitexin-2"-Orhamnoside on (a) cell viability and (b1 and b2) intracellular radical scavenging activities in HT-29 cells. Data are reported as the mean \pm SD of three replicates.

ing activity $\left(\mathrm{IC}_{50}=0.06 \mathrm{mg} / \mathrm{ml}\right)$ relative to total polyphenols $\left(\mathrm{IC}_{50}=0.15 \mathrm{mg} / \mathrm{ml}\right)$. A 4-OH group on B-ring is important for antioxidants, while the $5-\mathrm{OH}$ and $7-\mathrm{OH}$ groups on A-ring could bind to transition metals to form complexes, such as $\mathrm{Fe}^{2+}$, to block the reaction of Fenton ${ }^{23}$. Hence vitexin-2"O-rhamnoside exhibited high scavenging activity of hydroxyl radicals.

The results showed that the total antioxidant activity increased rapidly with increasing sample concentration (Fig. 3e), and the polyphenol content was more pronounced than that of vitexin-2"-Orhamnoside. This may be due in part to total polyphenols, which are a mixture of all antioxidant components such as rutin and quercetin-3-Oglucopyranoside. Their pyrocatechol structure on the B-ring increases the antioxidant activity of the total polyphenols ${ }^{24}$.
The DPPH scavenging activity increased as the concentration of total polyphenols and vitexin2"-O-rhamnoside increased from $0.04-1.0 \mathrm{mg} / \mathrm{ml}$ (Fig. 3f). The $\mathrm{IC}_{50}$ values were $0.32 \mathrm{mg} / \mathrm{ml}$ and $0.31 \mathrm{mg} / \mathrm{ml}$, respectively. These results indicate that there is no significant difference in DPPH free radical scavenging capacity between total polyphenols and vitexin-2"-O-rhamnoside solution in the range of $0.04-1.0 \mathrm{mg} / \mathrm{ml}$.

Cytotoxicity and anti-oxidative intracellular effects of polyphenolic compounds were evaluated in HT-29 cells. The results showed that there was no significant difference in HT-29 cell viability between total polyphenols and vitexin-2"-O-rhamnoside at different concentrations. Fig. 4a shows that they had no cytotoxic effect until the test concentration was $800 \mu \mathrm{g} / \mathrm{ml}$.

For the intracellular free radical scavenging activities (Fig. 4b1 and Fig. 4b2), the average fluorescence intensity of the control group treated with $200 \mu \mathrm{mol} / 1$ of $\mathrm{H}_{2} \mathrm{O}_{2}$ was almost eight times that of the blank. Total polyphenols and vitexin-2"O-rhamnoside significantly reduced average fluorescence intensity compared to the control group. The CAA value of total polyphenols increased from 39.47 to 86.39 , while the vitexin-2"-O-rhamnoside increased from 21.50 to 74.12. At the same concentration, the value of total polyphenols was higher than vitexin-2"-O-rhamnoside (by $t$-test, $p<0.05$ ). Vitexin-2"-O-rhamnoside had high activity for scavenging ROS in cells, attributed to the carbon 2, 3-double bond on the B-ring of the vitexin-2"-Orhamnoside structure, which may extend the conjugated system and significantly protect cells against $\mathrm{H}_{2} \mathrm{O}_{2}$-induced damage ${ }^{25}$.

Acknowledgements: This work was supported by the Tianjin Natural Science Foundation (grant No. 16JCYBJC43300, 17JCQNJC02400 and 18JCYBJC96000) and national undergraduate innovation and entrepreneurship education programs (grant No. 201810069023).

\section{REFERENCES}

1. Wang GW, Hu WT, Huang BK, Qin LP (2011) Illicium verum: a review on its botany, traditional use, chemistry, and pharmacology. J Ethnopharmacol 136, 10-20.

2. Bao NM, Dong XJ, Zhou L (2012) Chemical constitution from the twigs and leaves of Illicium verum Hook. J Northwest Agric Forestry Univ (Nat Sci Ed) 9, 231-234.

3. Yi GF, Long YX, Chen QB (2013) The determination of flavonoid aglycones in the leaves of Illicium verum Hook. Light Ind Sci Technol 7, 19-20. 
4. Sy LK, Brown GD (1998) Novel phenylpropanoids and lignans from Illicium verum. $J$ Nat Prod 61, 987-992.

5. Lu M, Yuan B, Zeng M, Chen J (2011) Antioxidant capacity and major phenolic compounds of spices commonly consumed in China. Food Res Int 44, 530-536.

6. Yang LC, Li R, Tan J, Jiang ZT (2013) Polyphenolics composition of the leaves of Zanthoxylum bungeanum Maxim. grown in Hebei, China, and their radical scavenging activities. J Agric Food Chem 61, 1772-1778.

7. Cao SL, Zhan HY, Fu SY, Chen LH (2007) Regulation of superoxide anion radical during the oxygen delignification process. Chin J Chem Eng 15, 132-137.

8. Ben-Nasr S, Aazza S, Mnif W, Miguel MDGC (2015) Antioxidant and antilipoxygenase activities of extracts from different parts of Lavatera cretica L. grown in Algarve (Portugal). Pharmacogn Mag 11, 48-54.

9. Liu J (2005) Research on a new method of determination and elimination of free radicals. $J$ Wuhan Polytech Univ 24, 53-55.

10. Young L, Sung J, Stacey G, Masters JR (2010) Detection of mycoplasma in cell cultures. Nat Protoc $\mathbf{5}$, 929-934.

11. Schwarz K, Bertelsen G, Nissen LR, Gardner PT, Heinonen MI, Hopia A, Huynh-Ba T, Lambelet P, et al (2001) Investigation of plant extracts for the protection of processed foods against lipid oxidation. Comparison of antioxidant assays based on radical scavenging, lipid oxidation, and analysis of the principal antioxidant compounds. Eur Food Res Technol 212, 319-328.

12. Zhu S, Yan H, Niu K, Zhang S (2015) Simultaneous determination of seven components from hawthorn leaves flavonoids in rat plasma by LC-MS/MS. $J$ Chromatogr Sci 53, 909-914.

13. Ma G, Jiang XH, Chen Z, Ren J, Li CR, Liu TM (2007) Simultaneous determination of vitexin4"-O-glucoside and vitexin-2"-O-rhamnoside from hawthorn leaves flavonoids in rat plasma by HPLC method and its application to pharmacokinetic studies. J Pharm Biomed Anal 44, 243-249.

14. Clifford MN, Wu W, Kuhnert N (2006) The chlorogenic acids of Hemerocallis. Food Chem 95, 574-578.

15. Gluchoff-Fiasson K, Jay M, Viricel MR (1989)
Flavone O-and C-glycosides from Setaria italica. Phytochemistry 28, 2471-2475.

16. Milbury PE, Chen CY, Dolnikowski GG, Blumberg JB (2006) Determination of flavonoids and phenolics and their distribution in almonds. J Agric Food Chem 54, 5027-5033.

17. Llorach R, Gil-Izquierdo A, Ferreres F, TomásBarberán FA (2003) HPLC-DAD-MS/MS ESI characterization of unusual highly glycosylated acylated flavonoids from cauliflower (Brassica oleracea $L$. var. botrytis) agroindustrial byproducts. J Agric Food Chem 51, 3895-3899.

18. Tanoli SAK, Tanoli NU, Bondancia TM, Usmani S, Ul-Haq Z, Fernandes JB, Thomasi SS, Ferreira AG (2015) Human serum albumin-specific recognition of the natural herbal extract of Stryphnodendron polyphyllum through STD NMR, hyphenations and docking simulation studies. RSC Adv 5, 23431-23442.

19. Sokkar NM, Rabeh MA, Ghazal G, Slem AM (2014) Determination of flavonoids in stamen, gynoecium, and petals of Magnolia grandiflora L. and their associated antioxidant and hepatoprotection activities. Quím Nova 37, 667-671.

20. Kim JE, Kin WY, Kim JW, Park HS, Lee SH, Lee SY, Kim MJ, Kim AR, et al (2010) Antibacterial, antioxidative activity and component analysis of Pinus koraiensis leaf extracts. J Soc Cosmet Sci Korea 36, 303-314.

21. Cholbi MR, Paya M, Alcaraz MJ (1991) Inhibitory effects of phenolic compounds on $\mathrm{CCl}_{4}$-induced microsomal lipid peroxidation. Experientia 47, 195-199.

22. Elumalai N, Ayyakkannu P, Palanivelu S, Panchanadham S (2015) In vitro antioxidant potential of Shemamruthaa (an herbal formulation) and its anticancer activity in the MCF-7 cell line. RSC Adv 5, 23125-23133.

23. Khokhar S, Apenten RKO (2003) Iron binding characteristics of phenolic compounds: Some tentative structure-activity relations. Food Chem 81, 133-140.

24. Record IR, Dreosti IE, McInerney JK (1995) The antioxidant activity of genistein in vitro. $J$ Nutr Biochem 6, 481-485.

25. Wang H, Joseph JA (1999) Structure-activity relationships of quercetin in antagonizing hydrogen peroxide-induced calcium dysregulation in PC12 cells. Free Radic Biol Med 27, 683-694. 